\title{
Open Fixation of Displaced Greater Tuberosity Fractures Yields Similar Patient-Reported Outcomes to Acute Arthroscopic Rotator Cuff Repair at 2 Years: A Matched Cohort Analysis
}

\author{
Dylan R. Rakowski, B.S., Joseph J. Ruzbarsky, M.D., Thomas E. Woolson, B.A., \\ Marilee P. Horan, M.P.H., Philip-C. Nolte, M.D, M.A., and Peter J. Millett, M.D., M.Sc.
}

\begin{abstract}
Purpose: To evaluate and compare patient-reported outcomes (PROs) after isolated greater tuberosity (GT) fracture fixation versus acute rotator cuff repair (RCR) at a minimum of 2 years. Methods: Patients who underwent isolated GT fracture fixation were compared in a l-to-3 fashion with patients who underwent arthroscopic RCR for an acute rotator cuff tear by a single surgeon from January 2006 and to July 2018. Data were prospectively collected and retrospectively reviewed. PROs were compared pre- and postoperatively as well as between groups (American Shoulder and Elbow Surgeons [ASES], General Health Short Form-12 Physical Component [SF-12 PCS], Single Assessment Numerical Evaluation [SANE], Quick Disabilities of the Arm, Shoulder, and Hand [QuickDASH], and satisfaction). Reoperation rates were analyzed. Results: A total of 57 patients (14 with isolated GT fracture fixation, mean age 45.7 years; and 43 who underwent ARCR for acute tears, mean age 56.6 years) were evaluated $(P=.050)$. ASES scores significantly improved from 39.7 to $94.1(P=.018)$ in the isolated GT fracture fixation group and from 51.0 to $95.2(P<.001)$ in acute RCR group. At final follow-up, mean QuickDASH scores were 8.9 and $7.9(P=.677)$ and SANE scores were 91.1 and $87.3(P=.616)$ for the GT and acute RCR groups, respectively. The median satisfaction was 10/10 for the GT group and 10/10 for the RCR group. Additional comparison of patients who underwent double-row repair for an acute rotator cuff tear or isolated GT fracture revealed no significant difference in outcomes $(P>.404)$. Conclusion: Minimum 2-year PROs after fixation of isolated GT fractures show relatively high outcome scores whether treated by open reduction and internal fixation or arthroscopic fixation using a double-row bridging technique. The improvements in PROs are similar to those achieved with acute rotator cuff tears that were fixed arthroscopically with RCR. Further analysis of these results suggest that the functional outcomes of tendon-to-bone healing with linked, double-row rotator cuff repairs are similar to those of boneto-bone healing as seen with GT fractures. Level of Evidence: III, retrospective comparative study.
\end{abstract}

$\mathbf{I}$ solated greater tuberosity (GT) fractures account for approximately one fifth of all proximal humerus fractures. $^{1,2}$ Although proximal humerus fractures are generally seen in elderly patients with osteoporosis

From the Steadman Philippon Research Institute, Vail, Colorado, U.S.A (D.R.R., J.J.R., T.E.W., M.P.H., P.J.M.); the Steadman Clinic, Vail, Colorado, U.S.A. (J.J.R., P.J.M.); and the Department of Trauma and Orthopedic Surgery, BG Trauma Center Ludwigshafen, Ludwigshafen, Germany (P.C.N.).

The authors report the following potential conflicts of interest or sources of funding: P.J.M. reports grants, personal fees, and other, Arthrex; personal fees, Springer Publishing, Medbridge; other, VuMedi, ProofPoint Biologics, Steadman Philippon Research Institute, Smith $\theta$ Nephew, Siemens, Össur. D.R.R. reports other, Arthrex, Smith $\theta$ Nephew, Siemens, Össur. J.J.R. reports personal fees, Steadman Philippon Research Institute. T.E.W. reports other, Arthrex, Smith $\theta$ Nephew, Siemens, Össur. M.P.H. reports other, Arthrex, Smith $\theta$ Nephew, Siemens, Össur. Full ICMJE author disclosure forms are available for this article online, as supplementary material. after a low-energy fall, isolated GT fractures are often seen in middle-aged patients after a glenohumeral dislocation or trauma to the lateral shoulder. ${ }^{3-6}$ Nondisplaced or minimally displaced fractures can be

Presentations: This project was presented at ISAKOS 2021 Global Congress, Nov. 27-28, 2021.

Received June 1, 2021; accepted December 21, 2021.

Address correspondence to Peter J. Millett, M.D., M.Sc., Center for Outcomes-based Orthopaedic Research, Steadman Philippon Research Institute, 181 West Meadow Dr, Suite 1000, Vail, CO 81657, U.S.A. E-mail: drmillett@thesteadmanclinic.com

(C) 2022 THE AUTHORS. Published by Elsevier Inc. on behalf of the Arthroscopy Association of North America. This is an open access article under the CC BY-NC-ND license (http://creativecommons.org/licenses/by-nc-nd/4.0/). 2666-061X/21761

https://doi.org/10.1016/j.asmr.2021.12.013 
treated effectively with nonoperative management, whereas surgery is generally indicated for fractures with $>5 \mathrm{~mm}$ of displacement or $>3 \mathrm{~mm}$ of displacement in athletes and individuals who perform work overhead. ${ }^{7,8}$ Operative management of these fractures allows for restoration of normal anatomy and aims to prevent subacromial impingement, pain, and shoulder dysfunction. ${ }^{9}$

Surgical management depends on the individual fracture characteristics and surgical skill with arthroscopy. Typically, severely displaced large bony fracture fragments are more commonly treated with open reduction and internal fixation, whereas comminuted fractures of the rotator cuff footprint are more likely to be approached arthroscopically and treated analogously to a rotator cuff repair. ${ }^{3}$ The arthroscopic approach to fracture fixation offers several advantages, including less surgical morbidity, adhesions, blood loss, and soft tissue trauma. ${ }^{10,11}$ Additionally, considering that $15 \%$ to $30 \%$ of glenohumeral dislocations have been reported to have a concomitant GT fracture, arthroscopic approaches allow surgeons to address any other pathologies such as Bankart lesions or rotator cuff tears. ${ }^{12}$ Stemming from the evolution of shoulder arthroscopy and rotator cuff repair, double-row fixation has been increasingly used for fracture fixation. ${ }^{13-16}$ Biomechanical analysis of the double-row technique has found that suture anchors restore the normal anatomy of the rotator cuff and exhibit a stronger initial fixation compared with screw fixation. ${ }^{17}$ Even with the prevalence of these fractures, there remains a paucity of research regarding the outcomes of GT fracture fixation.

The purpose of this study was to evaluate and compare patient-reported outcomes (PROs) after isolated GT fracture fixation to acute rotator cuff repair (RCR) at a minimum of 2 years. It is hypothesized that patients who underwent fixation of GT fractures with a double-row technique would have equivalent PRO measures to those treated for an acute rotator cuff tear.

\section{Methods}

Approval was granted by the institutional review board, Vail Health Hospital Institutional Review Board Protocol \#2020-29. All patients who underwent arthroscopic or open fixation of a GT fracture or RCR for an acute rotator cuff tear (defined as surgery $<7$ weeks since injury) performed by a single sports medicine fellowship-trained orthopaedic surgeon (P.J.M.) from January 2006 to July 2018 were included. Patients were excluded from the study if they did not have an isolated GT fracture or acute rotator cuff tear, did not undergo surgical fixation, or were out of country without contact information; if the date of injury could not be confirmed; or if the surgery was not within 7 weeks of injury. Preoperative diagnoses of greater tuberosity fractures and rotator cuff tears were made by clinical examination, x-ray, and magnetic resonance imaging (MRI) with attention to fracture comminution, fracture displacement, and concomitant pathologies. Preoperative diagnosis of acute rotator cuff tears was made by clinical examination and MRI with attention to tear type, tendon retraction, muscle atrophy, fatty infiltration (Goutallier et al. ${ }^{18} /$ Fuchs et al. ${ }^{19}$ classification), and concomitant pathologies. Surgery for both was indicated through joint decision making with the patient to treat persistent pain, loss of strength, and impaired function of the affected arm. Preoperatively, all patients consented to undergo either surgical fixation of a GT fracture or RCR, depending on the diagnosis.

\section{Surgical Technique}

\section{Linked, Double-Row Repair of a GT Fracture}

All surgeries were performed by a single surgeon (P.J.M.). After standard posterolateral and anterior portals were established, diagnostic arthroscopy was performed to inspect the glenohumeral joint and visualize the greater tuberosity fracture. If necessary, debridement of degenerative tissue or labral repair was performed. At this point, if a bicep tenodesis was indicated, the bicep tendon was released from its insertion on the superior labrum. Afterward, any subacromial pathology was addressed with the arthroscope in the subacromial space and through a working portal that was established $2 \mathrm{~cm}$ lateral from the anterolateral corner of the acromion. Next, attention was turned to the GT fracture.

After identification of the fracture, rotator cuff integrity was evaluated, and the mobility of the fracture fragment was assessed using a rotator cuff grasping device to ensure adequate rotator cuff excursion and fracture reduction. Next, the fracture site was debrided down to a bleeding bed with an arthroscopic burr and shaver to remove any blood clot or early callus. Intraoperatively, fluoroscopy was used to confirm proper anatomic reduction of the fracture fragment. Once confirmed, double-row repairs involved the placement of 2 medial row anchors. An arthroscopic punch was used to create a bone socket approximately 1 to $2 \mathrm{~mm}$ lateral to the articular surface of the humeral head. A 4.75-mm knotless anchor loaded with suture tape was placed in the anteromedial position, and a curved suture shuttling device was used to penetrate the rotator cuff tendon just medial to the GT fracture and then to pass the tape suture from the suture anchor from inferior to superior through the rotator cuff tendon. This procedure was repeated for the $4.75-\mathrm{mm}$ anchor placed in the posteromedial row position.

Before placement of the lateral row of anchors, an arthroscopic punch was used to create a bone socket 
approximately $5 \mathrm{~mm}$ lateral to the edge of the fracture site. One limb of the suture tape from each of the medial anchors was retrieved through the anterolateral portal and loaded into the eyelet of the anchor. The anchor was placed in the anterolateral position first and screwed into bone after appropriate tensioning. This same process was repeated for the posterior lateral row anchor to achieve compression of the fracture fragment. Intraoperative fluoroscopy was routinely used to confirm adequacy of reduction, and dynamic examination under anesthesia was used to assess both stability and impingement-free motion. In certain circumstances when the GT fracture was large in an anterior to posterior dimension, additional medial and lateral row anchors were used in the same configuration for an extended, linked double-row repair.

\section{Linked, Double-Row RCR}

All surgeries were performed by a single surgeon (P.J.M.) whose preferred technique has previously been described. ${ }^{20}$ After standard posterior and anterior portals were established, diagnostic arthroscopy was performed to inspect the glenohumeral joint. If necessary, debridement of degenerative tissue was performed.

The arthroscope was placed into the subacromial space to perform a subacromial decompression with acromioplasty when indicated. The acromion was contoured to a smooth flat margin in 2 planes using a modified cutting-block technique. Once the coracoacromial ligament was released, a normal scapulohumeral motion interface was reestablished, and a complete bursectomy was performed.

The linked, double-row rotator cuff repair was completed using 4 to 8 anchors depending on the severity of the rotator cuff tear. In the case of a 4anchor repair, first the GT was prepared, and the cuff tissue was debrided. Next, 2 anchors were placed medially, and using the same method of suture shuttling as described for the GT fracture above, the suture tapes from the medial row anchors were passed through the rotator cuff tendon. One suture tape from each medial row anchor was then placed into the eyelet of the lateral row anchor before anchor deployment to allow for compression of the tissue across the anatomic rotator cuff footprint.

\section{Postoperative Rehabilitation}

Postoperatively, patients undergoing GT fracture arthroscopic reduction and internal fixation were placed in a sling for comfort with limited passive range of motion (ROM) for 4 to 6 weeks (forward flexion $90^{\circ}$ to $120^{\circ}$, external rotation to $30^{\circ}$, abduction $90^{\circ}$ to $120^{\circ}$, internal rotation to body). At that point, full passive ROM was permitted, and the patient began activeassisted ROM for another 2 to 3 weeks while weaning from the brace. Once active-assisted shoulder movements were performed pain-free, the patient was cleared for active movement. At 8 to 10 weeks postoperatively, strengthening was initiated. If patients underwent a biceps tenodesis, passive and active elbow flexion were initiated immediately, but resistance elbow flexion was avoided for the first 6 weeks. Patients were cleared for full activities beginning at 4 to 5 months.

Postoperatively, RCR patients were placed in a brace for comfort with full passive ROM for 5 to 6 weeks. Depending on concomitant procedures, the passive ROM could be limited for the first 2 to 3 weeks. At 5 to 6 weeks, the patients were able to wean from the brace and begin working on full active ROM. At 6 weeks, patients started working on strengthening the shoulder while continuing to work on active ROM. Patients were cleared for full activities beginning at 4 to 5 months.

\section{Radiographic Assessment for GT Fracture Patients}

Radiographic evaluation included plain radiographs in anteroposterior, lateral, and axillary views. Preoperative imaging evaluation included number of fracture segments, fracture displacement, and fracture angulation. Postoperative radiographs were evaluated for bone union at the site of repair.

\section{Subjective Outcome Assessment/PROs}

Demographic data was collected including age, sex, days from injury to surgery, and concomitant procedures performed. Preoperatively, postoperatively at 6 months, and then annually, General Health Short Form-12 physical component ${ }^{21}$ (SF-12 PCS), American Shoulder and Elbow Surgeons ${ }^{22}$ (ASES) score, Single Assessment Numerical Evaluation ${ }^{23}$ (SANE) score, Quick Disabilities of the Arm, Shoulder, and Hand ${ }^{24}$ (QuickDASH) score, and patient satisfaction (on a 1to- 10 scale, with 10 being the best) were collected. Of note, our institution did not routinely collect SANE and QuickDASH until 2010, so preoperative scores were limited. The minimal clinically important difference (MCID), substantial clinical benefit (SCB), and patient acceptable symptom state (PASS) were used for the ASES score outcome parameters ${ }^{25}$ : 11.1, 17.5, and 86.7, respectively, for ASES scores.

\section{Reoperations and Complications}

Any complications or clinical failures were reported. Clinical failure was defined as the need for revision surgery or fracture nonunion.

\section{Statistical Analysis}

Univariate analyses were performed using an independent $t$ test for normally distributed variables. MannWhitney or Fisher exact tests were performed for data that were not normally distributed or bivariate comparison. Bivariate statistical analysis was used to address 
Table 1. Patient demographics

\begin{tabular}{lllc}
\hline Characteristic & Greater Tuberosity Fracture Fixation & Acute Rotator Cuff Repair & $P$ Value \\
\hline Males:females & $11: 3$ & $32: 11$ & 1.00 \\
Age (y) & $48.5(23.7$ to 73.6$)$ & $56.6(23.6$ to 72.8$)$ & .050 \\
Follow-up (y) & $6.3(2.1$ to 11.4$)$ & $7.0(2.1$ to 13.0$)$ & .468 \\
Days from injury to surgery & $16.1(1$ to 39$)$ & $20.4(1-45)$ & .317 \\
\hline
\end{tabular}

Data are average (range).

the primary aim of group comparisons between the acute RCR and GT fracture fixation groups. The chisquared test was used to assess relationships between 2 categorical variables. The Mann-Whitney $U$ test test was also used to compare PROs between groups, and the Wilcoxon signed-rank test was used to compare baseline and postoperative scores. Statistical power for this study was considered with respect to the known fixed sample size and the planned analysis for the primary endpoint. Assuming a 2-tailed Mann-Whitney $U$ test to compare independent group averages and an $\alpha$ of 0.05 , group sizes of 12 and 39 are sufficient to detect an effect size of Cohen's $d=0.97$ with $80 \%$ statistical power, where $d$ is the ratio of the difference in group means divided by the standard deviation. Thus, we conclude that between-group effect sizes $<0.97$ may not be ruled out by this study in the event of non-statistically significant group comparisons. Level of significance for univariate, paired $t$ tests, Wilcox rank-sum, categorical comparisons was set at $P<.05$. All statistical analyses were performed using SPSS version 11.0 (SPSS, Chicago, IL).

\section{Results}

\section{Study Population and Demographics}

From January 2006 to July 2018, a total of 87 patients with displaced GT fractures were identified. Of them, 72 patients were omitted due to concomitant fractures $(\mathrm{n}=58)$, nonoperative treatment $(\mathrm{n}=7)$, nonacute fixation $(n=2)$, inability to confirm the date of injury $(n=5)$, or status as out of the country and unable to be contacted $(\mathrm{n}=1)$ (Fig 1). This resulted in 14 total patients with isolated GT facture fixations who underwent outcomes analysis.

For acute rotator cuff repair, 225 patients were identified from January 2006 to July 2018. Of them, 182 were omitted as their surgery was $>6$ weeks after injury $(\mathrm{n}=177)$ or the date of injury could not be confirmed via chart review $(\mathrm{n}=5)$ (Fig 1). The remaining 43 patients who sustained acute, traumatic rotator cuff tears were compared in a 3:1 ratio to those who with GT fractures treated during the same time period. Minimum 2-year follow-up was obtained in 12 of 14 patients $(85.7 \%)$ with GT fractures and 39 of 43 $(90.6 \%)$ with acute rotator cuff repair . Demographic data for these 2 groups are detailed in Table 1. Average time to surgery from initial injury was 16.1 days (range 1 to 39 ) in the GT fracture fixation group and 20.4 days (range 1 to 45$)$ in the acute RCR group $(P=.317)$. In the GT fracture fixation group, 9 of the 14 patients underwent double-row bridging suture anchor repair fixation ( 5 arthroscopic and 4 open) of an isolated GT fracture and underwent a subgroup analysis to compare outcomes of double-row repair in the acute RCR group. The remaining patients underwent fixation via cannulated screws $(\mathrm{n}=3)$ or anchors not in double-row construct $(\mathrm{n}=2)$ (Fig 1). Outcomes were available in 9 of 9 patients $(100 \%)$ who underwent double-row bridging suture anchor repair at a minimum of 2 years after GT fracture fixation.

\section{PROs}

Preoperative and postoperative SF-12 PCS and ASES scores are detailed in Table 2. There was no difference between groups in preoperative SF-12 PCS $(P=.414)$ or ASES $(P=.173)$ scores. Between the groups, the improvement in PROs was significant for all measures except SF-12 PCS in the GT fixation group $(P=.110)$ (Table 2). In the isolated GT fracture fixation group, the ASES score exceeded the PASS threshold in 10 of 11 patients, and 6 of 6 patients exceeded the established MCID and SCB thresholds of 11.1 and 17.5 , respectively. ${ }^{25}$ In the acute rotator cuff repair group, the ASES score exceeded the PASS threshold in 34 of 36 patients. The MCID of 11.1 was achieved in 25 of 25 patients, and the SCB threshold

Table 2. Patient-reported outcomes (PROs)

\begin{tabular}{|c|c|c|c|c|c|c|}
\hline PRO & \multicolumn{3}{|c|}{ GT Fixation } & \multicolumn{3}{|c|}{ Acute RCR } \\
\hline$\overline{S F-12 ~ P C S ~}$ & 45.1 (32.2 to 58.3$)$ & 53.4 (40.6 to 57.9$)$ & .110 & 42.3 (28.8 to 56.7$)$ & $52.0(25.2$ to 61.9$)$ & $<.001$ \\
\hline
\end{tabular}

ASES, American Shoulder and Elbow Surgeons score; GT, greater tuberosity; RCR, rotator cuff repair; SF-12 PCS, General Health Short Form12 Physical Component. 
Table 3. Postoperative patient-reported outcomes (PROs)

\begin{tabular}{lccc}
\hline PRO & GT Fixation & Acute RCR & $P$ Value \\
\hline Post SF-12 PCS & $53.4(40.6$ to 57.9$)$ & $52.0(25.2$ to 61.9$)$ & 0.965 \\
Post ASES total & $94.1(80.0$ to 99.9$)$ & $95.2(49.9$ to 100$)$ & 0.172 \\
Post SANE & $91.1(49$ to 100$)$ & $87.3(19$ to 100$)$ & 0.616 \\
Post QuickDASH & $8.9(0$ to 25$)$ & $7.9(0$ to 45.4$)$ & 0.677 \\
Patient & $10(5$ to 10$)$ & $10(1$ to 10$)$ & 0.884 \\
$\quad$ & & &
\end{tabular}

ASES, American Shoulder and Elbow Surgeons score; GT, greater tuberosity; QuickDASH, Quick Disabilities of the Arm, Shoulder, and Hand; RCR, rotator cuff repair; SF-12 PCS, General Health Short Form-12 Physical Component.

of 17.5 for ASES score was exceeded in 24 of 25 patients. ${ }^{25}$ There were no significant differences found in PROs between groups at final follow-up (Table 3).

\section{Subgroup Analysis}

Double-Row Repairs

Between the groups, the change in PROs was significant for all measures except SF-12 PCS in the GT fixation group $(P=.176)$ and SANE in the RCR group $(P=.068)$ (Table 4$)$. The ASES scores exceeded the PASS threshold in 8 of 9 patients, and the MCID and SCB were exceeded in 6 of 6 patients who underwent GT fracture fixation using a double-row linked construct. Double-row repair in acute rotator cuff tears exceeded the ASES threshold for PASS in 32 of 34 patients, MCID in 23 of 23 patients, and SCB in 22 of 23 patients. There was no difference among the postoperative PROs between groups when double-row repairs were performed (Table 5).

\section{Complications and Revision Surgery}

Two patients $(14.3 \%)$ in the GT fracture fixation group reported stiffness postoperatively, both of whom had undergone open procedures with reduction and internal fixation. One patient was doing well for approximately 3.8 years until he felt a pop in his shoulder and developed acute pain, stiffness, and shoulder weakness. The patient received a corticosteroid injection and underwent a course of physical therapy without additional follow-up. One patient in the acute RCR group had recurrent shoulder pain and subsequently underwent revision surgery.

\section{Discussion}

The most important finding of this study is that linked, double-row repairs for isolated GT fractures yield excellent PROs at a minimum of 2 years postoperatively and that the results are similar to those of patients who have undergone acute RCR. Use of the acute RCR group allowed for a comparison of the outcomes associated with tendon-to-bone healing to that of bone-to-bone healing seen with GT fractures. Our study found that both groups significantly improved from preoperative baseline scores and that there were no significant differences between the groups' postoperative SF-12 PCS, ASES, SANE, QuickDASH, or satisfaction, and both exceeded the MCIB, SCB, and PASS thresholds for ASES score in a majority of patients. These results suggest that the functional outcomes of tendon-to-bone healing as seen with linked, double-row rotator cuff repairs may be similar to those of bone-to-bone healing as seen with GT fractures. Overall, this study highlights the importance of selecting a treatment-arthroscopic or open-based on fracture morphology but also postoperative goals, lifestyle, and shared-decision making for isolated GT fractures.

The literature contains several reports examining GT fracture fixation using double-row techniques. ${ }^{14,15,26}$ Bhatia et al. ${ }^{14}$ investigated open reduction and internal fixation using double-row suture anchor fixation for comminuted, displaced fractures of the GT. At a mean of 3.5 years, outcomes were excellent in 8 patients, good in 10, and satisfactory in 2, and 1 patient had an unsatisfactory result, but no ASES scores were available to compare with the results from our study. ${ }^{14}$ A study by Ji et al. ${ }^{15}$ evaluated 16 patients who underwent arthroscopic fixation of isolated comminuted, displaced GT fractures using double-row suture anchor fixation. At a mean of 24 months, postoperative visual analog pain scores improved from 9.4 to 1.2 , with final ASES and UCLA scores of 90.7 and 31, respectively. ${ }^{15}$ These results were comparable to the postoperative ASES score of 93.5 and significant improvement in the QuickDASH score from 65.4 to 8.1 in the current study. A previous study ${ }^{26}$ did compare open fixation with a locking plate to double-row fixation using suture

Table 4. Double-row subgroup: Patient-reported outcomes (PROs)

\begin{tabular}{|c|c|c|c|c|c|c|c|c|}
\hline \multirow[b]{2}{*}{ PRO } & \multicolumn{4}{|c|}{ Double-Row } & \multicolumn{4}{|c|}{ Not Double-Row } \\
\hline & Preoperative & Final Follow-Up & Change & $P$ Value & Preoperative & Final Follow-Up & Change & $P$ Value \\
\hline SF-12 PCS & 45.2 (33.8 to 58.3$)$ & 52.6 (40.6 to 57.9$)$ & +7.4 & .176 & 44.9 (32.2 to $57 . X)$ & $56.4(25.2$ to 61.9$)$ & +9.3 & $<<.001$ \\
\hline ASES total & $47.7(30.0$ to 66.6$)$ & 93.5 (80.0 to 99.9$)$ & +45.8 & .028 & $51.6(9.9$ to 83.3$)$ & $97.7(92$ to 100$)$ & +43.4 & $<0.001$ \\
\hline SANE & $35.4(0$ to 84$)$ & $90.8(49$ to 100$)$ & +55.4 & .043 & $49.5(10$ to 74$)$ & 95.2 (83 to 99$)$ & +37.1 & .068 \\
\hline QuickDASH & 65.4 (54.5 to 77.2$)$ & $8.1(0$ to 25.0$)$ & -57.3 & .039 & $45.2(31.8$ to 72.7$)$ & $7.5(0$ to 18.1$)$ & -37.1 & .002 \\
\hline
\end{tabular}

ASES, American Shoulder and Elbow Surgeons score; FFU, final Follow-up; GT, greater tuberosity; QuickDASH, Quick Disabilities of the Arm, Shoulder, and Hand; RCR, rotator cuff repair; SF-12 PCS, General Health Short Form-12 Physical Component. 
Table 5. Double-row subgroup: Postoperative patientreported outcomes (PROs)

\begin{tabular}{lccc}
\hline PRO & Double-Row & Not Double-Row & $P$ Value \\
\hline Post SF-12 PCS & $51.8(25.2$ to 61.9$)$ & $56.4(52.8$ to 60$)$ & 0.404 \\
Post ASES total & $94.6(50.0$ to 100$)$ & $97.7(91.6$ to 100$)$ & 0.675 \\
Post SANE & $87.2(19$ to 100$)$ & $95.2(83$ to 99$)$ & 0.864 \\
Post QuickDASH & $8.2(0$ to 45.4$)$ & $7.5(0$ to 18.1$)$ & 0.913 \\
Patient & $10(1$ to 10$)$ & 10 (range 1 to 10$)$ & 0.834 \\
$\quad$ Satisfaction & & &
\end{tabular}

ASES, American Shoulder and Elbow Surgeons score; GT, greater tuberosity; QuickDASH, Quick Disabilities of the Arm, Shoulder, and Hand; RCR, rotator cuff repair; SF-12 PCS, General Health Short Form-12 Physical Component.

anchors and found that patients who underwent double-row repair with suture anchor fixation had a longer operating time but better improvement in shoulder forward flexion and abduction and higher ASES scores. The importance of this clinical difference in ASES score between the groups is questionable, however, as it does not meet the MCID threshold of 11.1 points between the groups. ${ }^{25,26}$ Overall, the group undergoing GT fracture fixation in the current study had relatively high outcome scores whether they were treated by open reduction and internal fixation or arthroscopic fixation, which relays the importance of selecting a treatment based on fracture morphology but also postoperative goals, lifestyle, and shared decisionmaking.

The current study also evaluated all patients who underwent surgical fixation of a GT fracture regardless of technique and found a significant improvement in ASES score from 39.7 to 94.1, in which all patients exceeded the MCID and SCB thresholds, although 2 patients did experience postoperative stiffness. A recent systematic review by Huntley et al. ${ }^{27}$ included 16 studies and consisted of 345 patients who underwent surgical fixation of isolated greater tuberosity fractures. The mean follow-up in this study was quite short at 3.4 months, but the ASES score was 90.1 and DASH was 12.8, which are comparable to the ASES score of 94.1 and QuickDASH of 8.9 found in this study. ${ }^{27}$ Yoon et al. ${ }^{28}$ investigated minimally invasive open reduction and internal fixation by screw and washer in a cohort of 29 patients. At the 2-year follow-up point, patients exhibited a mean ASES score of 92.6, but 9 patients $(31 \%)$ underwent subsequent arthroscopic release owing to pain and stiffness. ${ }^{28}$ Although no patient in the GT group underwent additional surgery, $25 \%$ of patients who underwent open fixation did report modifying activity postoperatively because of stiffness. Although the numbers are small, arthroscopic techniques may reduce the risk of postoperative stiffness owing to a better ability to therapeutically lavage the joint of marrow elements that are present in the setting of a fracture which, when present in the subacromial, subdeltoid, and glenohumeral spaces, may lead to adhesions and decreased range of motion. The current study adds to the sparse research regarding patientreported outcomes after isolated greater tuberosity fracture fixation and allows for a comparison of the outcomes associated with tendon-to-bone healing to that of bone-to-bone healing seen with GT fractures.

Further research should focus on whether arthroscopy with debridement has benefit during cases of planned open reduction and internal fixation in an attempt to reduce postoperative stiffness. Additional research comparing the various techniques of greater tuberosity fractures should be compared to delineate patient outcomes with these methods.

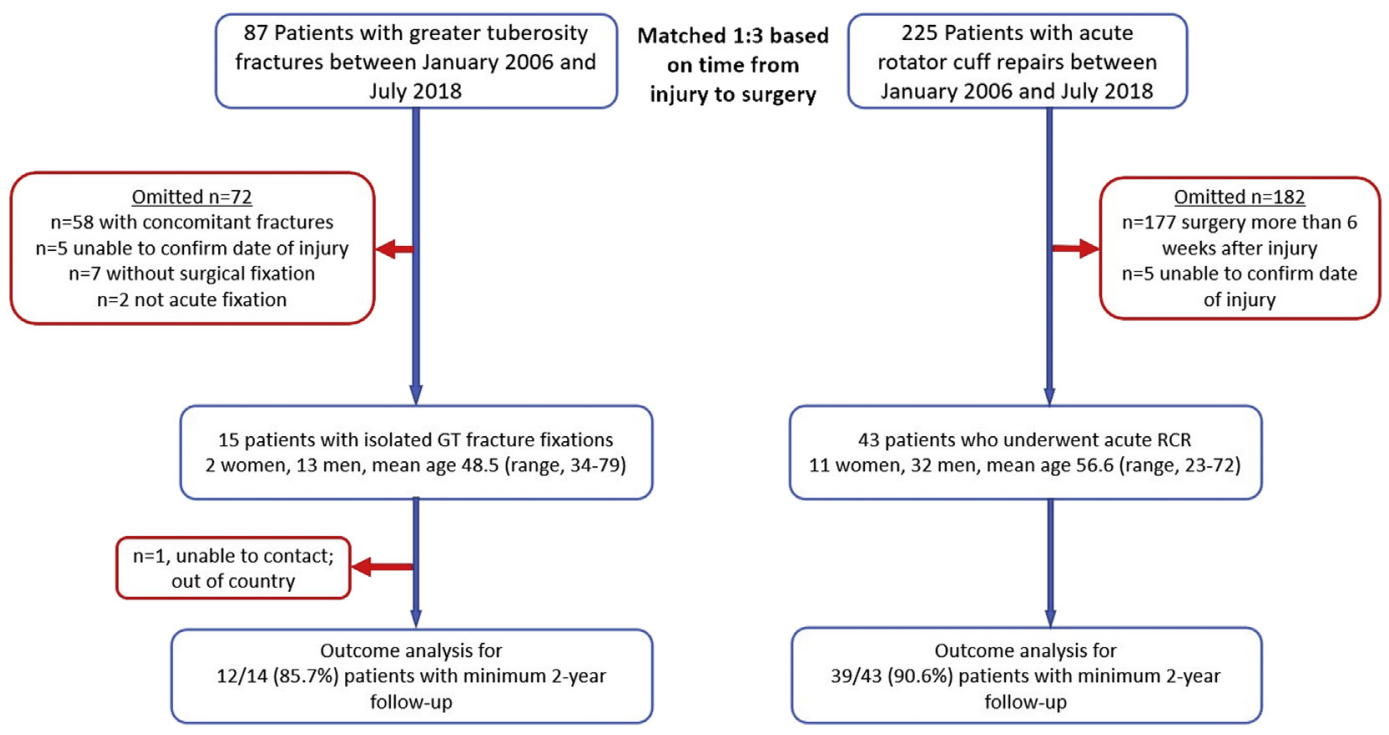

Fig 1. Study flow diagram. Abbreviations: GT, greater tuberosity; RCR, rotator cuff repair. 


\section{Limitations}

This study has several limitations, the most significant being the small size of the GT cohort and the heterogeneity of interventions in the treatment group. Given the various options present for surgical fixation, patients were consulted on the various treatment approaches and methods, and shared decision-making was agreed on between physician and patient, taking into account the individual patient's timeline of injury, goals, lifestyle, and fracture characteristics. Although this is a limitation, we believe it is important to report overall outcomes of this group given the paucity of published data on GT fracture fixation. Another limitation of this study is the lack of SANE and QuickDASH scores that were available preoperatively. Our institution did not routinely collect SANE and QuickDASH until 2010; thus we were unable to report on pre- to postoperative improvement in the GT fracture fixation or acute RCR group as a whole. Finally, these results may not be generalizable to other populations, as our practice is a sports medicine referral center and our patients are generally healthy, with few comorbidities.

\section{Conclusions}

Minimum 2-year patient-reported outcomes after fixation of isolated GT fractures show relatively high outcome scores whether treated by open reduction and internal fixation or arthroscopic fixation using a double-row, bridging suture anchor technique. The improvements in PROs are similar to those achieved with acute rotator cuff tears that were fixed arthroscopically with RCR. Further analysis of these results suggests that the functional outcomes of tendon-tobone healing with linked, double-row rotator cuff repairs are similar to those of bone-to-bone healing as seen with GT fractures.

\section{References}

1. Kim E, Shin HK, Kim CH. Characteristics of an isolated greater tuberosity fracture of the humerus. J Orthop Sci 2005; 10:441-444.

2. Court-Brown CM, Garg A, McQueen MM. The epidemiology of proximal humeral fractures. Acta Orthop Scand 2001;72:365-371.

3. Gruson KI, Ruchelsman DE, Tejwani NC. Isolated tuberosity fractures of the proximal humerus: Current concepts. Injury 2008;39:284:198.

4. Fakler JK, Hogan C, Heyde CE, John T. Current concepts in the treatment of proximal humeral fractures. Orthopedics 2008;31:42-51.

5. Kim S-H, Ha K-I. Arthroscopic treatment of symptomatic shoulders with minimally displaced greater tuberosity fracture. Arthroscopy 2000;16:695-700.

6. Rouleau DM, Mutch J, Laflamme GY. Surgical treatment of displaced greater tuberosity fractures of the humerus. J Am Acad Orthop Surg 2016;24:46-56.
7. McLaughlin HL. Dislocation of the shoulder with tuberosity fracture. Surg Clin N Am 1963;43:1615-1620.

8. Park TS, Choi IY, Kim YH, Park MR, Shon JH, Kim SI, A new suggestion for the treatment of minimally displaced fractures of the greater tuberosity of the proximal humerus. Bull Hosp Joint Dis 1997;56:171-176.

9. Platzer P, Kutscha-Lissberg F, Lehr S, Vecsei V, Gaebler C. The influence of displacement on shoulder function in patients with minimally displaced fractures of the greater tuberosity. Injury 2005;36:1185-1189.

10. Taverna E, Sansone V, Battistella F. Arthroscopic treatment for greater tuberosity fractures: Rationale and surgical technique. Arthroscopy 2004;20:e53-e57.

11. Katthagen JC, Jensen G, Voigt C, Lill H. Arthroskopie bei proximaler Humerusfraktur. Arthroskopie 2014;27:265-274.

12. Robinson CM, Shur N, Sharpe T, Ray A, Murray IR. Injuries associated with traumatic anterior glenohumeral dislocations. J Bone Joint Surg Am 2012;94:18-26.

13. Godin JA, Katthagen JC, Fritz EM, Pogorzelski J, Millett PJ. Arthroscopic treatment of greater tuberosity avulsion fractures. Arthrosc Tech 2017;6:e777-e783.

14. Bhatia DN, van Rooyen KS, du Toit DF, de Beer JF. Surgical treatment of comminuted, displaced fractures of the greater tuberosity of the proximal humerus: A new technique of double-row suture-anchor fixation and long-term results. Injury 2006;37:946-952.

15. Ji JH, Shafi M, Song IS, Kim YY, McFarland EG, Moon CY. Arthroscopic fixation technique for comminuted, displaced greater tuberosity fracture. Arthroscopy 2010;26:600-609.

16. Park SE, Ji JH, Shafi M, Jung JJ, Gil HJ, Lee HH. Arthroscopic management of occult greater tuberosity fracture of the shoulder. Eur J Orthop Surg Traumatol 2014;24:475-482.

17. Lin C-L, Hong C-K, Jou IM, Lin C-J, Su F-C, Su W-R. Suture anchor versus screw fixation for greater tuberosity fractures of the humerus-a biomechanical study. J Orthop Res 2012;30:423-428.

18. Goutallier D, Postel JM, Bernageau J, Lavau L, Voisin MC. Fatty muscle degeneration in cuff ruptures. Pre- and postoperative evaluation by CT scan. Clin Orthop Relat Res 1994:78-83.

19. Fuchs B, Weishaupt D, Zanetti M, Hodler J, Gerber C. Fatty degeneration of the muscles of the rotator cuff: Assessment by computed tomography versus magnetic resonance imaging. J Shoulder Elbow Surg 1999;8:599-605.

20. Mook WR, Greenspoon JA, Millett PJ. Arthroscopic double-row transosseous equivalent rotator cuff repair with a knotless self-reinforcing technique. Open Orthop $J$ 2016;10:286-295.

21. Fan ZJ, Smith CK, Silverstein BA. Assessing validity of the QuickDASH and SF-12 as surveillance tools among workers with neck or upper extremity musculoskeletal disorders. J Hand Ther 2008;21:354-365.

22. Kocher MS, Horan MP, Briggs KK, Richardson TR, O’Holleran J, Hawkins RJ. Reliability, validity, and responsiveness of the American Shoulder and Elbow Surgeons subjective shoulder scale in patients with shoulder instability, rotator cuff disease, and glenohumeral arthritis. J Bone Joint Surg Am 2005;87:2006-2011.

23. Williams GN, Gangel TJ, Arciero RA, Uhorchak JM, Taylor DC. Comparison of the Single Assessment Numeric 
Evaluation method and two shoulder rating scales. Outcomes measures after shoulder surgery. Am J Sports Med 1999;27:214-221.

24. Beaton DE, Wright JG, Katz JN. Development of the QuickDASH: Comparison of three item-reduction approaches. J Bone Joint Surg Am 2005;87:1038-1046.

25. Cvetanovich GL, Gowd AK, Liu JN, Nwachukwu BU, Cabarcas BC, Cole BJ, et al. Establishing clinically significant outcome after arthroscopic rotator cuff repair. J Shoulder Elbow Surg 2019;28:939-948.

26. Liao W, Zhang H, Li Z, Li J. Is arthroscopic technique superior to open reduction internal fixation in the treatment of isolated displaced greater tuberosity fractures? Clin Orthop Relat Res 2016:474:1269-1279.

27. Huntley SR, Lehtonen EJ, Robin JX, Arguello AM, Rouleau DM, Brabston EW, et al. Outcomes of surgical fixation of greater tuberosity fractures: A systematic review. Orthop Traumatol Surg Res 2020;106: $1119-1126$.

28. Yoon TH, Choi CH, Choi YR, Oh JT, Chun YM. Clinical outcomes of minimally invasive open reduction and internal fixation by screw and washer for displaced greater tuberosity fracture of the humerus. J Shoulder Elbow Surg 2018;27:e173-e177. 